\title{
CARving up colorectal cancer organoids in vitro
}

\author{
Christian M. Schürch (i) $^{1}$
}

Received: 29 April 2019 / Accepted: 1 May 2019 / Published online: 20 May 2019

(c) Springer Nature Limited 2019. This article is published with open access

Colorectal cancer (CRC) is one of the leading causes of cancer morbidity and mortality worldwide [1]. Over the last decades, the introduction of screening tests, changes in lifestyle and risk factors, and advances in surgery and diagnostics have reduced CRC incidence and mortality. In addition, clinical outcomes of patients with metastatic disease (mCRC) have improved substantially, with median overall survival exceeding 30 months in the era of combination systemic chemotherapy, targeted therapies, and resection or ablation of localized metastases [2-4]. This is reflected by an increasing number of mCRC patients treated within the framework of clinical trials or specialized cancer centers, and is paralleled by the development of novel imaging modalities and molecular tests to monitor these patients. Nevertheless, unfortunately, the majority of mCRC patients are still not definitely cured and will ultimately succumb to their disease.

Chimeric antigen receptor (CAR) $\mathrm{T}$ cells have induced spectacular remissions and are now approved for use in patients with relapsed/refractory hematological malignancies. This has revived the interest in using CAR T cells and natural killer (NK) cells in solid tumors such as CRC, especially in the metastatic setting, and drives the need for the identification of antigen targets, and the development of preclinical models to test these therapies [5, 6]. One of the many important key prerequisites for CAR $\mathrm{T}$ cell and NK cell therapies to be effective in solid tumors is the ability of the CAR cells to penetrate into, and survive in, the tumor microenvironment (TME). The TME consists of vasculature, stroma, innate and adaptive immune cell subsets, and extracellular matrix (ECM), and is the primary location of tumor cell-immune cell interactions. In many solid tumors,

Christian M. Schürch

schuerch@stanford.edu

1 Baxter Laboratory for Stem Cell Biology, Department of Microbiology and Immunology, Stanford University School of Medicine, Stanford, CA, USA the TME is immunosuppressive by preventing effective recruitment of tumor-specific adaptive immune cells (chemokine mismatch, aberrant vasculature, and ECM barriers) and/or by preventing their survival and effector functions (expression of checkpoint receptor ligands, recruitment of regulatory $\mathrm{T}$ cells, myeloid-derived suppressor cells, etc.) [7]. Therefore, in order for CAR cell therapies to be improved and to ultimately succeed also in solid tumors, it is necessary to better understand these cells' interactions, mobility, survival, and effector functions in the context of the TME.

Writing in the EMBO Journal, Schnalzger et al. report an elegant in vitro platform for preclinical testing of CAR cells using patient-derived CRC organoids [8]. This is an important step toward the accomplishment of abovementioned goals and is well in line with ongoing efforts toward personalized cancer medicine. First, the authors modified the organoid culture conditions such that CAR NK cell killing can occur. Nicotinamide (vitamin B3), a supplement present in standard organoid culture media, was identified as an NK cell-inhibitory factor, and removing nicotinamide restored NK cell killing without affecting organoid viability. In addition, the authors found that culturing organoids on top of a matrigel scaffold, as compared to within the matrigel or in suspension, resulted in effective CAR NK cell-mediated organoid killing. The observation that epithelial cell adhesion molecule (EPCAM)-specific CAR NK cells were unable to kill normal colon organoids when these were cultured within the matrigel or in suspension indicates that CAR cells need an ECM scaffold to perform their function, probably for attachment and crawling, while simultaneously being unable to penetrate into this scaffold. This is an important finding that warrants future investigation to dissect the mechanisms of CAR cell-ECM interactions.

Second, the authors optimized their system by retrovirally transducing the organoids with a luciferase-GFP vector to monitor the extent of killing after different time points. On one hand, the introduction of luciferase eliminates the need for microscopic counting of organoids and 


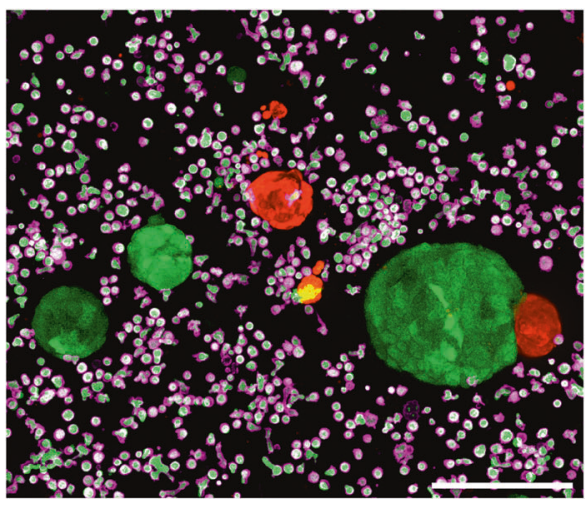

Fig. 1 Live microscopy of CRC organoid killing in vitro. EGFRvIIItransduced CRC organoids (GFP-positive, green) were co-cultured with normal colon organoids (DsRED-positive, red) and EGFRvIIIspecific CAR NK cells (GFP-positive, green) stained with anti-CD45-

significantly simplifies the experimental setup, allowing to collect and store lysed supernatants, and increasing the throughput of this platform. On the other hand, GFP expression, in conjunction with CD45 staining of NK cells, allows for live-cell microscopy-based visualization and quantification of killing at the single-organoid level. Importantly, both luciferase- and live-imaging-based experimental readouts yielded similar results.

The authors then expanded their model to two clinically relevant cancer-associated antigens, epidermal growth factor receptor, variant III (EGFRvIII) and FRIZZLED (FZD), the WNT receptor. EGFRvIII, a constitutively active EGFR mutant lacking exons $2-7$, is a neoantigen frequently amplified in solid tumors [9]. FZD is overexpressed in a subset of CRCs that lack RNF43/ZNRF3, a receptor that complexes with LGR-5 and negatively regulates WNT signaling by promoting FZD internalization and degradation in response to R-spondin [10]. EGFRvIII-specific CAR NK cells were highly selective toward organoids lentivirally transduced with this EGFR mutant, and no off-target toxicity was observed in a competitive killing assay in the presence of healthy colon organoids (Fig. 1). In contrast, FZD-specific CAR NK cells killed organoids regardless of their FZD receptor status, indicating that a clinical strategy targeting FZD would likely result in mucosal toxicity.

Although the study by Schnalzger et al. is an important step toward translating the preclinical testing of CAR cells into personalized cancer medicine, several key questions remain open. First, the results with FZD-targeting NK cells that also destroyed normal colon organoids emphasize that optimal antigenic targets for mCRC need to be identified. Recently, several clinical trials for CAR cell therapy of solid tumors such as prostate cancer (target: prostate-specific membrane antigen (PSMA)), mesothelioma, pancreatic and ovarian cancer (target: mesothelin), and glioma (targets:

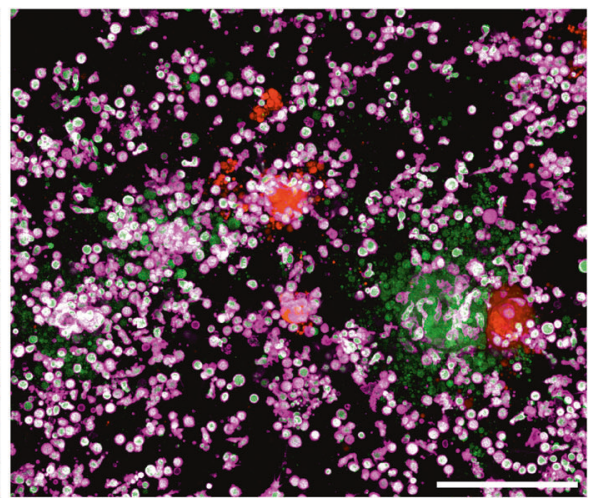

APC (magenta). Two different time points are shown (left panel, $0 \mathrm{~h}$; right panel, $10 \mathrm{~h}$ ). Maximal intensity projection images, scale bars $200 \mathrm{~mm}$. Images courtesy of Henner Farin, Goethe University Frankfurt

EGFR, EGFRvIII, and HER2) have been initiated [5]. For $\mathrm{CRC}$, preclinical models using CAR $\mathrm{T}$ cells directed against guanylyl cyclase C (GUCY2C), an enzyme overexpressed in CRC metastases, showed promising therapeutic activity, and a GUC2YC vaccination was well tolerated in 10 CRC patients without metastases (ClinicalTrials.gov NCT01972737) [1113]. An advantage of GUC2YC as a CAR target is that its expression is restricted to the apical (luminal) surface in normal epithelia, a feature that is lost in metastases, rendering them visible to the CAR cells. However, GUC2YC is also expressed by a subset of hypothalamic neurons, and neurotoxicity is therefore a caveat [13].

Second, the ideal effector-to-target (E:T) cell ratio has to be determined to allow for optimal tumor cell killing with minimal side effects in vivo. In their in vitro model, the authors observed increasing killing at E:T ratios starting from 0.5:1, with highest efficacy at 4:1. However, how these ratios translate into a clinical setting is still ill defined.

Third, the role of TME and ECM and their effects on CAR cell behavior must be better defined in order to understand and improve future CAR cell therapies for solid tumors. The study by Schnalzger et al. is an important step in this direction and their in vitro organoid killing platform paves the way to answer these questions in the future.

Acknowledgements C.M.S. was supported by an Advanced Postdoc Mobility Fellowship from the Swiss National Science Foundation (P300PB_171189).

\section{Compliance with ethical standards}

Conflict of interest The author declares that he has no conflict of interest.

Publisher's note: Springer Nature remains neutral with regard to jurisdictional claims in published maps and institutional affiliations. 
Open Access This article is licensed under a Creative Commons Attribution 4.0 International License, which permits use, sharing, adaptation, distribution and reproduction in any medium or format, as long as you give appropriate credit to the original author(s) and the source, provide a link to the Creative Commons license, and indicate if changes were made. The images or other third party material in this article are included in the article's Creative Commons license, unless indicated otherwise in a credit line to the material. If material is not included in the article's Creative Commons license and your intended use is not permitted by statutory regulation or exceeds the permitted use, you will need to obtain permission directly from the copyright holder. To view a copy of this license, visit http://creativecommons. org/licenses/by/4.0/.

\section{References}

1. Bray F, Ferlay J, Soerjomataram I, Siegel RL, Torre LA, Jemal A. Global cancer statistics 2018: GLOBOCAN estimates of incidence and mortality worldwide for 36 cancers in 185 countries. CA Cancer J Clin. 2018;68:394-424.

2. Fakih MG. Metastatic colorectal cancer: current state and future directions. J Clin Oncol. 2015;33:1809-24.

3. Van Cutsem E, Cervantes A, Adam R, Sobrero A, Van Krieken $\mathrm{JH}$, Aderka D, et al. ESMO consensus guidelines for the management of patients with metastatic colorectal cancer. Ann Oncol. 2016;27:1386-422.

4. Siegel RL, Miller KD, Fedewa SA, Ahnen DJ, Meester RGS, Barzi A, et al. Colorectal cancer statistics. CA Cancer J Clin. 2017;67:177-93.
5. Jackson HJ, Rafiq S, Brentjens RJ. Driving CAR T-cells forward. Nat Rev Clin Oncol. 2016;13:370-83.

6. D'Aloia MM, Zizzari IG, Sacchetti B, Pierelli L, Alimandi M. CAR-T cells: the long and winding road to solid tumors. Cell Death Dis. 2018;9:282.

7. Slaney CY, Kershaw MH, Darcy PK. Trafficking of T cells into tumors. Cancer Res. 2014;74:7168-74.

8. Schnalzger TE, de Groot MHP, Zhang C, Mosa MH, Michels BE, Röder J, et al. 3D model for CAR-mediated cytotoxicity using patient-derived colorectal cancer organoids. EMBO J. 2019; e100928.

9. Gan HK, Cvrljevic AN, Johns TG. The epidermal growth factor receptor variant III (EGFRvIII): where wild things are altered. FEBS J. 2013;280:5350-70.

10. Giannakis M, Hodis E, Jasmine Mu X, Yamauchi M, Rosenbluh $\mathrm{J}$, Cibulskis K, et al. RNF43 is frequently mutated in colorectal and endometrial cancers. Nat Genet. 2014;46:1264-6.

11. Snook AE, Baybutt TR, Mastrangelo MJ, Lewis NL, Goldstein SD, Kraft WK, et al. A phase I study of AD5-GUCY2C-PADRE in stage I and II colon cancer patients [abstract]. 2015. https://jdc. jefferson.edu/petposters/2.

12. Magee MS, Kraft CL, Abraham TS, Baybutt TR, Marszalowicz GP, Li P, et al. GUCY2C-directed CAR-T cells oppose colorectal cancer metastases without autoimmunity. Oncoimmunology. 2016;5:e1227897.

13. Magee MS, Abraham TS, Baybutt TR, Flickinger JC Jr., Ridge NA, Marszalowicz GP, et al. Human GUCY2C-Targeted chimeric antigen receptor (CAR)-expressing $\mathrm{T}$ Cells eliminate colorectal cancer metastases. Cancer Immunol Res. 2018;6:509-16. 